\title{
Identification of key genes for predicting colorectal cancer prognosis by integrated bioinformatics analysis
}

\author{
GONG-PENG DAI $^{1 *}$, LI-PING WANG ${ }^{2 *}$, YU-QING WEN $^{1 *}$, XUE-QUN REN $^{1}$ and SHU-GUANG ZUO ${ }^{2,3}$ \\ ${ }^{1}$ Department of General Surgery; ${ }^{2}$ Center for Translational Medicine; ${ }^{3}$ Institute of Infection and Immunity, \\ Huaihe Hospital of Henan University, Kaifeng, Henan 475001, P.R. China
}

Received October 3, 2018; Accepted September 17, 2019

DOI: $10.3892 / 01.2019 .11068$

\begin{abstract}
Colorectal cancer (CRC) is a life-threatening disease with a poor prognosis. Therefore, it is crucial to identify molecular prognostic biomarkers for CRC. The present study aimed to identify potential key genes that could be used to predict the prognosis of patients with CRC. Three CRC microarray datasets (GSE20916, GSE73360 and GSE44861) were downloaded from the Gene Expression Omnibus (GEO) database, and one dataset was obtained from The Cancer Genome Atlas (TCGA) database. The three GEO datasets were analyzed to detect differentially expressed genes (DEGs) using the BRB-ArrayTools software. Functional and pathway enrichment analyses of these DEGs were performed using the Database for Annotation, Visualization and Integrated Discovery tool. A protein-protein interaction (PPI) network of DEGs was constructed, hub genes were extracted, and modules of the PPI network were analyzed. To investigate the prognostic values of the hub genes in CRC, data from the CRC datasets of TCGA were used to perform the survival analyses based on the sample splitting method and Cox regression model. Correlation among the hub genes was evaluated using Spearman's correlation analysis. In the three GEO datasets, a total of 105 common DEGs were identified, including 51 down- and 54 up-regulated genes in CRC compared with normal colorectal tissues. A PPI network consisting of 100 DEGs and 551 edges was constructed, and 44 nodes were identified as hub genes. Among these 44 genes, the four hub
\end{abstract}

Correspondence to: Dr Xue-Qun Ren, Department of General Surgery, Huaihe Hospital of Henan University, 115 Ximen Street, Kaifeng, Henan 475001, P.R. China

E-mail: hhyyrxq@126.com

Dr Shu-Guang Zuo, Center for Translational Medicine, Huaihe Hospital of Henan University, 115 Ximen Street, Kaifeng, Henan 475001, P.R. China

E-mail: zuosg@icloud.com

*Contributed equally

Key words: colorectal cancer, differentially expressed genes, protein-protein interaction, survival genes TIMP metallopeptidase inhibitor 1 (TIMP1), solute carrier family 4 member 4 (SLC4A4), aldo-keto reductase family 1 member B10 (AKR1B10) and ATP binding cassette subfamily E member 1 (ABCE1) were associated with overall survival (OS) in patients with CRC. Three significant modules were extracted from the PPI network. The hub gene TIMP1 was present in Module 1, ABCE1 was involved in Module 2 and SLC4A4 was identified in Module 3. Univariate analysis revealed that TIMP1, SLC4A4, AKR1B10 and ABCE1 were associated with the OS of patients with CRC. Multivariate analysis demonstrated that SLC4A4 may be an independent prognostic factor associated with OS. Furthermore, the results from correlation analysis revealed that there was no correlation between TIMP1, SLC4A4 and ABCE1, whereas AKR1B10 was positively correlated with SLC4A4. In conclusion, the four key genes TIMP1, SLC4A4, AKR1B10 and ABCE1 associated with the OS of patients with CRC were identified by integrated bioinformatics analysis. These key genes may be used as prognostic biomarkers to predict the survival of patients with CRC, and may therefore represent novel therapeutic targets for CRC.

\section{Introduction}

Colorectal cancer (CRC) is the third most common type of cancer, and the fourth leading cause of cancer associated-mortality in the world, with 1.8 million new cases diagnosed and 881,000 deaths in $2018(1,2)$. Since surgical techniques and chemotherapy regimen have been improved, and molecular targets have been determined, attention has focused on early detection of CRC, which led to an increase in survival time of patients with CRC $(3,4)$. However, the 5-year overall survival (OS) rate of patients with CRC is still unsatisfactory, particularly for patients with advanced CRC (5). In China, CRC is the third most frequent type of cancer and the fourth leading cause of cancer-associated mortality (6). In order to precisely predict the prognosis of patients with CRC, it is crucial to identify novel molecular prognostic biomarkers.

Many lifestyle factors have been investigated, with imbalanced diet, tobacco use, alcohol consumption, lack of physical activity, obesity and sleep deprivation being considered as the risk factors in the progression of CRC (7). Heredity, epigenetic, somatic cell and endocrine aberration seem to play significant roles in CRC (8). Increasing evidence has suggested that multiple genes and cellular signaling pathways serve 
important roles in the pathogenesis of CRC $(9,10)$. Diagnostic and prognostic signatures, for example BRAF, RAS and MSI, can monitor the response to therapy in patients with CRC (11). Therefore, it is critical to identify novel biomarkers with high sensitivity and specificity in order to allow the early detection of CRC and to choose the best treatment option for patients with CRC. Microarray analysis has been considered as a promising tool in cancer research and may have important clinical applications, including in diagnosis, cancer classification, prognosis prediction and detection of therapeutic targets (12-14). In the last decade, microarray technology has been used to study the gene expression profiles of CRC, which has led to the identification of thousands of differentially expressed genes (DEGs) (15). However, DEGs identified in one study might not be detected in another study. In addition, interactions among DEGs and their prognostic values remain unknown.

The present study aimed to determine potential novel prognosis biomarkers for CRC. Three microarray datasets obtained from the Gene Expression Omnibus (GEO) database were analyzed in order to further identify the DEGs in CRC, by comparing their expression levels between CRC and normal samples. Subsequently, a protein-protein interaction (PPI) network was constructed, from which modules were extracted, and functional and pathway analyses were performed to further analyze the roles of the CRC-associated DEGs. To verify the prognostic roles of DEGs in CRC, the CRC dataset from The Cancer Genome Atlas (TCGA) was used to perform survival analysis based on the sample splitting method and Cox regression model. The key genes that were identified may be used to characterize the survival of CRC, and may serve as potential therapeutic targets and prognostic biomarkers.

\section{Materials and methods}

Microarray datasets. The GEO database (http://www.ncbi. nlm.nih.gov/geo) is a public functional genomics data repository that contains multifaceted data, including data derived from microarray and next-generation sequencing. GEO database was searched using the following key words: ('colorectal neoplasms'[MeSH Terms] OR colorectal cancer [All Fields])) AND 'Homo sapiens'[porgn] AND ('gse'[Filter] AND 'Expression profiling by array'[Filter] AND 'attribute name tissue'[Filter] AND ('60’[n_samples] : ‘3000'[n_samples])) AND ('gse'[Filter] AND 'Expression profiling by array'[Filter] AND 'attribute name tissue'[Filter] AND ('60'[n_samples]: '3000'[n_samples])). Following systematic review based on the key words, a total of 77 datasets were identified for further analysis. The selection criteria for these datasets were as follows: i) Included datasets must include paired CRC and normal control tissues; ii) sample size of each group must be $>30$; and iii) adequate clinical information must be available to perform the analysis. According to the selection criteria, three gene expression profiles were collected, including GSE20916 (16), GSE73360 (17) and GSE44861 (18). The microarray data of GSE20916, GSE73360 and GSE44861 were downloaded from the GEO database. GSE20916 was based on the Affymetrix GPL570 platform (Human Genome U133 Plus 2.0 Array), GSE73360 was based on the Affymetrix GPL17586 platform (Human Transcriptome Array 2.0) and GSE44861 was based on Affymetrix GPL3921 platform (Human
Genome U133A Array). The GSE20916 dataset included 101 CRC tissue samples and 44 normal samples. Expression data from the GSE73360 dataset included 55 CRC samples and 37 normal samples. Microarray data from the GSE44861 dataset included 56 CRC samples and 55 normal samples.

Data pre-processing and identification of DEGs in CRC. The Series Matrix File(s) of GSE20916, GSE73360 and GSE44861 were downloaded from the GEO database. Prior to analysis, the probes in each dataset were transformed into standard gene symbols. Normalization of the three datasets was implemented based on robust multi-array average in the $\mathrm{R}$ software, version 2.6.0 (www.R-project.org/) (19), and normalization was separately conducted in each gene expression dataset.

The BRB-ArrayTools package (v4.6.0 Beta 1; https://brb. nci.nih.gov/BRB-ArrayTools/download.html) is an integrated software for the visualization and statistical analysis of microarray gene-expression data, gene-methylation data and RNA-sequencing data (20). In the present study, DEGs from each dataset were identified using BRB-ArrayTools software. A P-value $<0.01$ and llog fold changel $>1$ were set as the cut-off criteria. A heatmap and Volcano Plot of the DEGs from each dataset were subsequently generated using BRB-ArrayTools. The Venn diagram illustrating the intersection of these DEGs among the three microarray profiles was visualized using FunRich_V3.1.3 software (http://www.funrich.org).

PPI network construction and module analysis. The Search Tool for the Retrieval of Interacting Genes (STRING; https://string-db.org) database is an online software that is used to analyze PPI information, and to present the interactions with a combine-score (21). In the present study, DEGs with a confidence score $>0.4$ were extracted, and the PPI network of these DEGs was subsequently constructed and visualized using Cytoscape software (version 3.5.1; http://www.cytoscape.org) (22). Edge width was determined according to the combined score of the PPI relationship. Since the networks were scale-free, the biological importance of genes associated with degree centrality was described to improve the understanding of the functionality of these complicated networks. The degree is determined as the number of links between a node and its adjacent nodes (23). In the current study, hub genes were defined as the nodes with a degree $>10$, and were extracted.

The analysis of modules was conducted on the PPI network by using MCODE version 2.2 (https://dspace. mit.edu/handle/1721.1/75242) according to the following parameters: Node score cut-off $=0.2, \mathrm{k}$-core $=2$ and $\max$. Depth=100 (24).

Functional and pathway enrichment analysis. Gene Ontology (GO) (25) analysis has become a common method used for functional studies of large-scale transcriptomic data and analysis of genomic data. Kyoto Encyclopedia of Genes and Genomes (KEGG; http://www.genome.jp/kegg/pathway) is a reference database for systematic analysis of gene functions (26). The Database for Annotation Visualization and Integrated Discovery (DAVID; https://david.ncifcrf.gov) is a tool used to systematically detect biological significance in large lists of genes or proteins (27). In the present study, 
the GO function and KEGG pathway enrichment analysis of identified DEGs and the genes in the significant modules were performed using DAVID. Terms with a P-value $<0.001$ were considered statistically significant.

Pathway crosstalk analysis. Enrichment map is a network-based method for gene-set enrichment visualization and interpretation (28). In order to extract the interactions between significantly enriched signaling pathways, pathway crosstalk analysis was performed using the EnrichmentMap tool (http://www.baderlab.org/Software/EnrichmentMap). Benjamini-Hochberg adjusted P-value $<0.05$ and the Jaccard Coefficient $(50 \%)+$ Overlap Coefficient $(50 \%)>0.5$ were considered as the thresholds. The Jaccard Coefficient, and Overlap Coefficient are two indexes to examine the similarity between sample sets.

Survival analysis of hub genes. The Human Protein Atlas (http://www.proteinatlas.org) is an online database that contains clinical data of 597 patients with CRC, which were from the TCGA database (https://tcga-data.nci.nih. gov/tcga/). The present study aimed to investigate the impact of the expression levels of the identified hub genes on the OS of patients with CRC. Kaplan-Meier survival analysis was carried out by using the Human Protein Atlas database. The survival rates of patients with CRC according to the expression level of each hub gene were compared. Briefly, according to the Fragments Per Kilobase Million (FPKM) value of each hub gene, patients with CRC were classified into a high or low expression group based on the best expression cut-off value, which is the FPKM value that yields maximal difference by survival analysis between the two groups at the smallest log-rank P-value. Kaplan-Meier survival curves were plotted, and the log-rank test was applied to compare the survival rates between the high and low expression groups. $\mathrm{P}<0.001$ was set as the cut-off criterion. Univariate and multivariate Cox models were subsequently used to investigate the prognostic value of OS-associated hub genes. $\mathrm{P}<0.01$ was considered to indicate statistical significance.

Correlation between hub genes. The correlation between the hub genes that were associated with CRC was evaluated using Spearman's correlation analysis. Spearman's correlation coefficients range between -1 and 1 , with $-1,0$ and 1 indicating negative, no and positive correlation, respectively. In addition, the threshold for gene correlation was set as Spearman's correlation coefficient $(|\mathrm{R}|<0.3)$ and $\mathrm{P}<0.05$.

\section{Results}

Identification of DEGs. A total of 2,836, 2,942 and 420 DEGs were identified from the GSE20916, GSE73360 and GSE44861 datasets, respectively. Volcano plots showing the distribution of these DEGs in the three datasets were created (Fig. 1A). The results from cluster heat maps (Fig. 1B) identified distinctive patterns in CRC and normal samples. Subsequently, the intersection of these DEGs from the three microarray profiles was identified using FunRich_V3 software. A total of 105 mutual DEGs were identified in CRC tissues among the three datasets compared with normal colorectal tissues
(Fig. 1C), including 51 down-regulated and 54 up-regulated genes. Fig. 1D shows the expression pattern of the common up-regulated and down-regulated DEGs from the expression data of the GSE20916, GSE44861 and GSE73360 datasets.

Functional and pathway enrichment analysis. In order to comprehensively understand the biological roles of these DEGs in CRC, DAVID was used to determine the GO functions and pathways in which they were involved (Table I). The down-regulated DEGs were significantly enriched in the biological processes 'bicarbonate transport', and 'regulation of intracellular $\mathrm{pH}$ ', and in the cellular components 'extracellular exosome' and 'integral component of plasma membrane'. Furthermore, down-regulated DEGs were enriched in the molecular functions 'chloride channel activity' and 'carbonate dehydratase activity'. The up-regulated DEGs were significantly enriched in the biological processes 'cell proliferation', 'collagen catabolic process', 'extracellular matrix organization', 'extracellular matrix disassembly', and 'leukocyte migration' in certain cellular components, including 'extracellular space', 'extracellular region', 'proteinaceous extracellular matrix', 'collagen trimer' and 'cell surface', and in the molecular functions 'CXCR chemokine receptor binding', 'platelet-derived growth factor binding' and 'endopeptidase activity'.

The 20 significant pathways which the up- and down-regulated DEGs were involved in are presented in Table II. The results demonstrated that down-regulated DEGs were significantly enriched in the pathways 'proximal tubule bicarbonate reclamation' and 'nitrogen metabolism', whereas up-regulated DEGs were enriched in 'ECM-receptor interaction', 'focal adhesion', 'PI3K-Akt signaling pathway' and 'TNF signaling pathway'.

PPI network construction and module analysis. The PPIs of the DEGs were obtained using STRING with a confidence score $>0.4$, and the PPI network of these DEGs was visualized using Cytoscape. The results demonstrated that the PPI network covered 100 nodes and 551 edges, including 49 down-regulated genes and 51 up-regulated genes (Fig. 2A). Subsequently, hub nodes in the PPI network with a connectivity degree $>10$ were selected. A total of 44 genes were identified as hub genes. Among these hub genes, 17 were down-regulated [transmembrane serine protease 2 , sorcin, solute carrier family 4 member 4 (SLC4A4), solute carrier family 26 member 3, nuclear receptor subfamily 3 group $C$ member 2, myosin heavy chain 11, keratin 20, 15-hydroxyprostaglandin dehydrogenase, guanylate cyclase activator 2A, fatty acid binding protein 1, CEA cell adhesion molecule 7, carbonic anhydrase 4, carbonic anhydrase 2, carbonic anhydrase 1, ADP ribosylation factor like GTPase 14, alanyl aminopeptidase, membrane and aldo-keto reductase family 1 member B10 (AKR1B10)] and 27 were up-regulated [TIMP metallopeptidase inhibitor 1 (TIMP1), thrombospondin 2 , transforming growth factor $\beta$ induced, stanniocalcin 1 , secreted phosphoprotein 1 , secreted protein acidic and cysteine rich, serpin family B member 5, stearoyl-CoA desaturase, receptor interacting serine/threonine kinase 2, regenerating family member $3 \alpha$, prostaglandin-endoperoxide synthase 2 , MYC proto-oncogene, bHLH transcription factor, matrix metallopeptidase 3, matrix metallopeptidase 1, MET proto-oncogene, receptor tyrosine kinase, leucine rich repeat containing $\mathrm{G}$ 
A

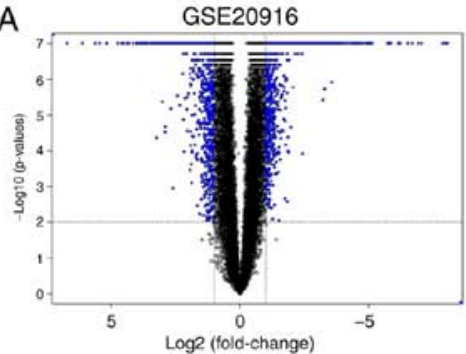

B

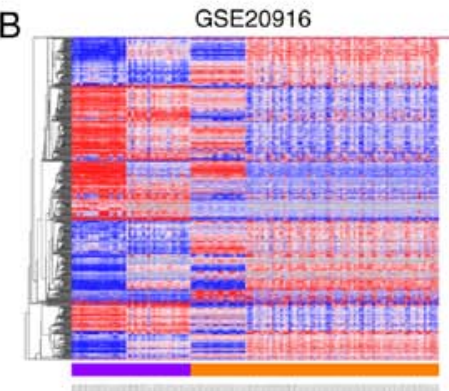

C

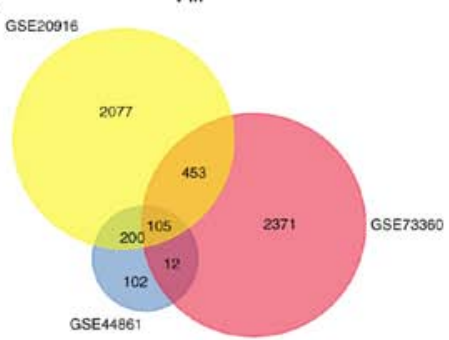

GSE44861

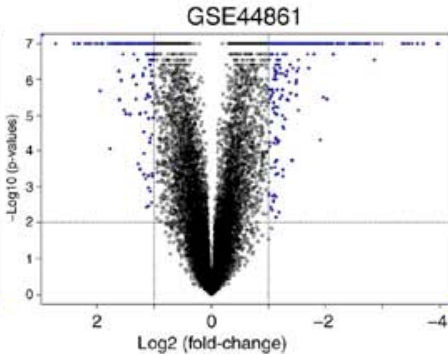

GSE44861

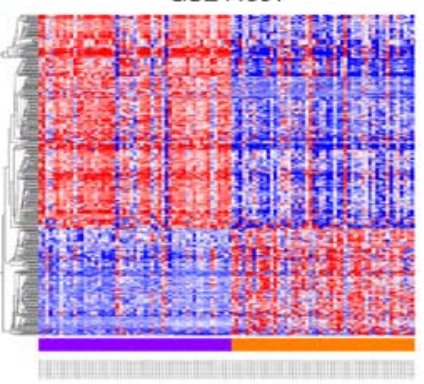

Down

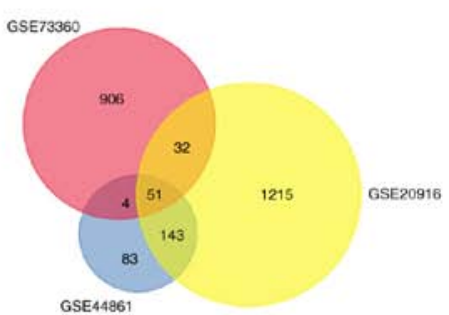

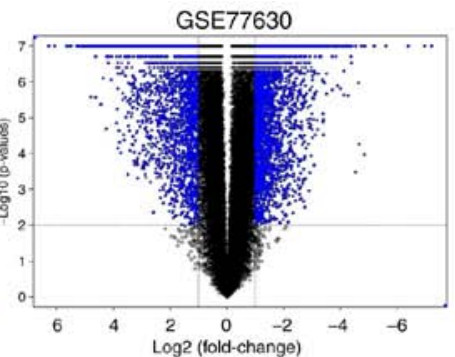

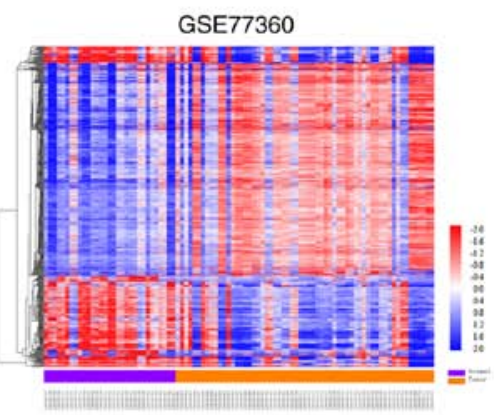

Up

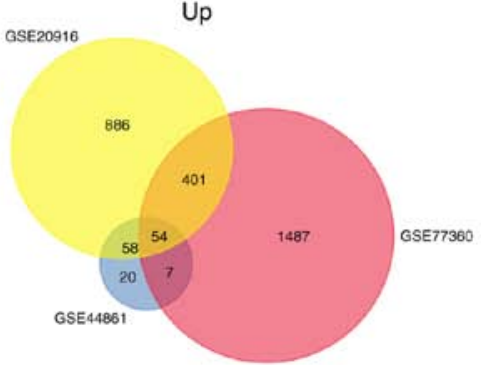

D
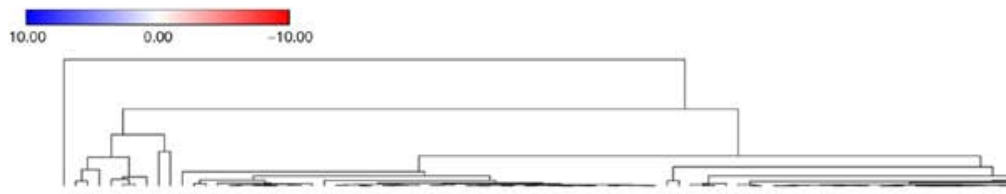

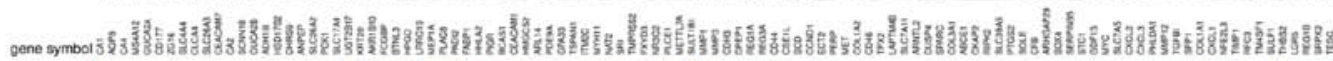

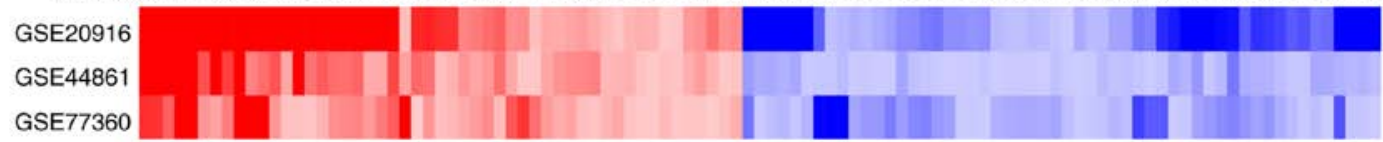

Figure 1. Expression pattern of genes between CRC and normal samples. (A) Volcano plots exhibiting expression data of CRC and normal tissues in the microarray profiles of GSE20916, GSE44861 and GSE73360. The x-axis presents the mean differences between CRC and normal samples. The y-axis presents the $\log$ transformed P-values. DEGs are shown in blue. (B) Hierarchical clustering analysis of DEGs between CRC and normal samples in GSE20916, GSE44861 and GSE77360. Each row represents a DEG and each column represents a different sample. (C) Intersection of all DEGs (n=105), up-regulated DEGs (n=54) and down-regulated DEGs ( $\mathrm{n}=51$ ) among the expression data of GSE20916, GSE44861 and GSE73360. (D) Hierarchical clustering analysis of the mutual up-regulated and down-regulated DEGs in the expression data of GSE20916, GSE44861 and GSE73360. Red represents down-regulated genes, whereas blue represents up-regulated genes. CRC, colorectal cancer; DEGs, differentially expressed genes.

protein-coupled receptor 5 , growth differentiation factor 15 , dual specificity phosphatase $4, \mathrm{C}-\mathrm{X}-\mathrm{C}$ motif chemokine ligand 2, C-X-C motif chemokine ligand 1, collagen type III $\alpha 1$ chain, collagen type I $\alpha 2$ chain, collagen type I $\alpha 1$ chain, complement factor B, CD44 molecule (Indian blood group), cyclin D1 and ATP binding cassette subfamily E member 1 (ABCE1)].

Three significant modules with a node score cut-off $=0.2$, $\mathrm{k}$-core $=2$, and max. Depth $=100$ were extracted from the PPI network. A total of 18 nodes and 136 edges were included in Module 1 (Fig. 2B). There were six nodes and 12 edges in Module 2 (Fig. 2C), and Module 3 contained six nodes and 10 edges (Fig. 2D). The up-regulated hub gene TIMP1 was included in Module 1, the up-regulated ABCE1 gene was involved in Module 2 and the down-regulated SLC4A4 was present in Module 3.

Module 1 was enriched in eight GO terms, including 'extracellular matrix organization', 'extracellular space' and 'platelet-derived growth factor binding'. Module 2 was enriched in two GO terms; 'ATPase activity' and 'protein binding'. Module 3 was enriched in the four terms 'regulation of intracellular pH', 'bicarbonate transport', 'chloride transmembrane transport' and 'chloride channel activity' (Table III).

In addition, the results from KEGG pathway enrichment analysis demonstrated that genes in Module 1 were significantly 
Table I. GO analysis of differentially expressed genes in colorectal cancer tissues.

A, Downregulated

\begin{tabular}{|c|c|c|c|c|}
\hline Category & Term & Count, $\mathrm{n}$ & Percentage & P-value \\
\hline \multirow[t]{2}{*}{ GOTERM_BP_DIRECT } & GO:0015701 'bicarbonate transport' & 6 & 9.17 & $1.10 \times 10^{-7}$ \\
\hline & GO:0051453 'regulation of intracellular $\mathrm{pH}$ ' & 4 & 6.11 & $1.21 \times 10^{-4}$ \\
\hline \multirow[t]{2}{*}{ GOTERM_CC_DIRECT } & GO:0070062 'extracellular exosome' & 29 & 44.32 & $2.28 \times 10^{-11}$ \\
\hline & GO:0005887 'integral component of plasma membrane' & 15 & 22.95 & $1.38 \times 10^{-5}$ \\
\hline \multirow[t]{2}{*}{ GOTERM_MF_DIRECT } & GO:0005254 'chloride channel activity' & 4 & 6.11 & $4.54 \times 10^{-4}$ \\
\hline & GO:0004089 'carbonate dehydratase activity' & 3 & 4.59 & $6.76 \times 10^{-4}$ \\
\hline
\end{tabular}

B, Upregulated

\begin{tabular}{|c|c|c|c|c|}
\hline Category & Term & Count, $\mathrm{n}$ & Percentage & P-value \\
\hline \multirow[t]{5}{*}{ GOTERM_BP_DIRECT } & GO:0030574 'collagen catabolic process' & 6 & 7.70 & $1.55 \times 10^{-6}$ \\
\hline & GO:0030198 ‘extracellular matrix organization’ & 8 & 10.26 & $2.27 \times 10^{-6}$ \\
\hline & GO:0022617 'extracellular matrix disassembly’ & 6 & 7.70 & $3.66 \times 10^{-6}$ \\
\hline & GO:0050900 ‘leukocyte migration’ & 6 & 7.70 & $3.69 \times 10^{-5}$ \\
\hline & GO:0008283 'cell proliferation' & 8 & 10.26 & $1.27 \times 10^{-4}$ \\
\hline \multirow[t]{5}{*}{ GOTERM_CC_DIRECT } & GO:0005615 'extracellular space' & 19 & 24.38 & $2.07 \times 10^{-8}$ \\
\hline & GO:0005576 ‘extracellular region’ & 17 & 21.81 & $8.07 \times 10^{-6}$ \\
\hline & GO:0005578 'proteinaceous extracellular matrix' & 7 & 9.00 & $1.23 \times 10^{-4}$ \\
\hline & GO:0005581 'collagen trimer' & 5 & 6.42 & $1.47 \times 10^{-4}$ \\
\hline & GO:0009986 ‘cell surface’ & 8 & 10.26 & $9.33 \times 10^{-4}$ \\
\hline \multirow[t]{3}{*}{ GOTERM_MF_DIRECT } & GO:0045236 ‘CXCR chemokine receptor binding’ & 3 & 3.85 & $3.05 \times 10^{-4}$ \\
\hline & GO:0048407 'platelet-derived growth factor binding' & 3 & 3.85 & $4.65 \times 10^{-4}$ \\
\hline & GO:0004175 ‘endopeptidase activity’ & 4 & 5.13 & $5.45 \times 10^{-4}$ \\
\hline
\end{tabular}

$\mathrm{BP}$, biological process; $\mathrm{CC}$, cellular component; GO, Gene Ontology; MF, molecular function.

involved in the four pathways 'ECM-receptor interaction', 'PI3K-Akt signaling pathway', 'focal adhesion' and 'miRNAs in cancer'. The genes in Module 3 were significantly enriched in the 'pancreatic secretion' pathway (Table IV).

Pathway crosstalk analysis. To determine how the 20 significant pathways interacted with each other, a pathway crosstalk analysis was conducted. The results demonstrated that a cross talk existed among 'PI3K-Akt signaling pathway', 'focal adhesion' and 'ECM-receptor interaction'. In addition, a separate crosstalk existed between 'proximal tubule bicarbonate reclamation' and 'nitrogen metabolism'. No interaction was observed between 'TNF signaling pathway' and the other pathways (Fig. 3).

Survival analysis of hub genes. The prognostic value of the 44 hub genes from the PPI network was investigated using The Human Protein Atlas database. The results demonstrated that four hub genes were associated with the OS of patients with CRC, including TIMP1, SLC4A4, AKR1B10 and ABCE1 (Fig. 4). The results of the survival analysis suggested that TIMP1 may be considered as an oncogene, whereas SLC4A4, AKR1B10 and ABCE1 may be tumor suppressor genes.
The results from univariate analysis revealed that the Union for International Cancer Control (UICC) stage (29) and TIMP1, SLC4A4, AKR1B10 and ABCE1 expression levels were significantly associated with the OS of patients with CRC (all $\mathrm{P}<0.01$ ). However, there was no significant association between age/sex and OS in the univariate analysis $(\mathrm{P}>0.01)$. The results of multivariate analysis demonstrated that age, UICC stage and SLC4A4 expression were independent prognostic factors associated with the OS of patients with CRC (Table $\mathrm{V}$; all $\mathrm{P}<0.01$ ).

Correlation between hub genes. Correlation analysis between the expression levels of the four hub genes (TIMP1, SLC4A4, ABCE1 and AKR1B10) was performed using Spearman's correlation analysis. The results demonstrated that there was no correlation between TIMP1, SLC4A4 and ABCE1 expression (Fig. 5); however, the expression levels of AKR1B10 and SLC4A4 were positively correlated $(\mathrm{R}=0.39 ; \mathrm{P}<0.05$; Fig. 5).

\section{Discussion}

Despite advances in surgical and medical therapies for the treatment of $\mathrm{CRC}$, the incidence and mortality rates remain 
Table II. Kyoto Encyclopedia of Genes and Genomes pathway analysis of differentially expressed genes in colorectal cancer tissues.

A, Downregulated

\begin{tabular}{lcccc}
\hline Term & Count, $\mathrm{n}$ & Percentage & P-value & Genes \\
\hline $\begin{array}{l}\text { hsa04964: Proximal tubule bicarbonate } \\
\text { reclamation }\end{array}$ & 4 & 6.11 & $7.06 \times 10^{-5}$ & CA4, CA2, SLC4A4, PCK1 \\
hsa00910: Nitrogen metabolism & 3 & 8.25 & $5.12 \times 10^{-4}$ & CA2, CLCA4, SLC4A4 \\
\hline
\end{tabular}

B, Upregulated

\begin{tabular}{|c|c|c|c|c|}
\hline Term & Count, $\mathrm{n}$ & Percentage & P-value & Genes \\
\hline hsa04512: ECM-receptor interaction & 6 & 7.70 & $1.83 \times 10^{-5}$ & $\begin{array}{l}\text { CD } 44, \text { COL } 3 \text { A } 1, \text { COL1A2, COL1A1, } \\
\text { THBS2, SPP1 }\end{array}$ \\
\hline hsa04510: Focal adhesion & 7 & 9.00 & $1.14 \times 10^{-4}$ & $\begin{array}{l}\text { CCND1, COL3A1, MET, COL1A2, } \\
\text { COL1A1, THBS2, SPP1 }\end{array}$ \\
\hline hsa04151: PI3K-Akt signaling pathway & 8 & 10.26 & $2.71 \times 10^{-4}$ & $\begin{array}{l}\text { CCND1, COL3A1, MET, COL1A2, } \\
\text { COL1A1, THBS2, MYC, SPP1 }\end{array}$ \\
\hline hsa04668: TNF signaling pathway & 5 & 6.42 & $7.00 \times 10^{-4}$ & $\begin{array}{l}\text { CXCL1, PTGS2, CXCL3, CXCL2, } \\
\text { MMP3 }\end{array}$ \\
\hline
\end{tabular}

ECM, extracellular matrix; TNF, tumor necrosis factor.

high $(1,2)$. Successful screening techniques and reducing the risk of CRC are essential to help decrease the incidence of CRC. Understanding the etiology and mechanisms of CRC progression is crucial to improve the survival rate of patients with CRC and prevent the disease occurrence. Recently, microarray technology, which has rapidly developed, has been widely used to compare the expression levels of genes, and has been used to predict disease progression, to make an accurate diagnosis and evaluate prognosis (30-32). In the present study, a meta-analysis method was used to analyze three microarray datasets (GSE20916, GSE73360 and GSE44861) in order to identify DEGs in CRC tissue samples. A total of 105 mutual DEGs were identified in the three datasets, including 51 down-regulated genes and 54 up-regulated genes. A total of 44 DEGs were subsequently selected from the PPI network and were identified as hub genes. Furthermore, three significant modules were identified in the PPI network. In addition, survival analysis of the hub genes demonstrated that two down-regulated genes (SLC4A4 and AKR1B10) and two up-regulated genes (ABCE1 and TIMP1) were significantly associated with the OS of patients with CRC. These four genes may be the most reliable genes that could be applied in clinical settings for the following reasons: i) These four genes were simultaneously confirmed using three gene expression profile datasets; and ii) these genes have been demonstrated to be associated with cancer.

SLC4A4, which is a member of the SLC4 family, is an electrically induced transmembrane transporter, which is mostly involved in sodium and bicarbonate transport to the epithelial cell membrane (33). A previous study reported that the SLC4 family is mainly involved in $\mathrm{CO}_{2}$ transport by red blood cells, the absorption or secretion of $\mathrm{H}^{+}$or $\mathrm{HCO}^{-}$by epithelial cells and regulation of cell volume and intracellular $\mathrm{pH}$ in the majority of cells (34). Furthermore, abnormal SLC4A4 expression has been reported in thyroid carcinoma, and this biomarker may be used for the diagnosis of thyroid cancer (35). However, to the best of our knowledge, only a few studies have investigated SLC4A4 expression in CRC. For example, Chen et al (36) demonstrated that the expression of SLC4A4 was decreased in CRC samples. Another study also reported that SLC4A4 was significantly down-regulated in the CRC group (37). In the present study, a PPI network was constructed to identify the hub genes for CRC, and SLC4A4 was identified to be down-regulated. The clinical data of 597 patients with CRC were then collected using TCGA database. The results from survival analysis demonstrated that patients with CRC in the SLC4A4 high expression group had a longer OS compared with patients with CRC in the SLC4A4 low expression group. These findings suggested that SLC4A4 may be used to evaluate the prognosis of patients with CRC.

ABCE1 is located on chromosome $4 \mathrm{q} 31$ and codes for 599 amino acids. Previous studies have reported that ABCE1 suppresses the interferon (IFN)-dependent 2-5A/RNase L system and serves key roles in cell proliferation and apoptosis $(38,39)$. IFN is involved in cell immune defense by inducing transcription processes of a large number of genes, and has been demonstrated to be able to fight virus infection, inhibit tumor growth and control cell proliferation and differentiation (39). Zheng et al (40) have demonstrated that $\mathrm{ABCE} 1$ is up-regulated in lung cancer samples, and that decreased ABCE1 expression can impair cell proliferation and increase cell apoptosis. Hlavata et al (41) also reported that ABCE1 is up-regulated in CRC. Furthermore, it has been reported that ABCE1 knockdown can inhibit the 

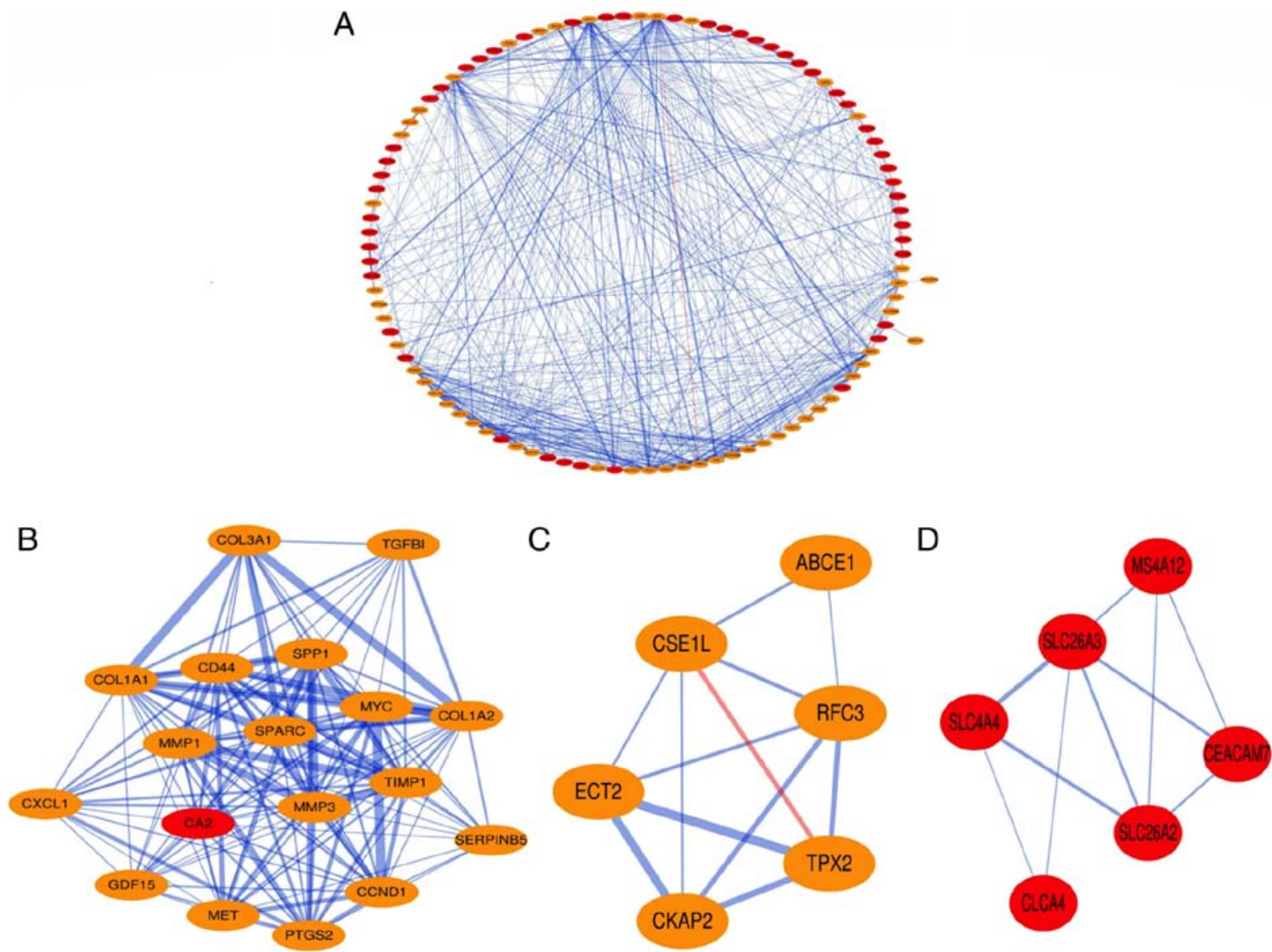

Figure 2. PPI network construction and module composition. (A) Construction of the PPI network covered 100 nodes and 551 edges, including 49 up-regulated genes and 51 down-regulated genes. The nodes represent the protein (gene). Red nodes represent down-regulated DEGs. Orange nodes represent up-regulated DEGs. Edge width was determined according to the combined score of the PPI relationship. (B) A total of 18 nodes and 136 edges were included in Module 1. (C) A total of six nodes and 12 edges were included in Module 2. (D) A total of six nodes and 10 edges were included in Module 2. DEGs, differentially expressed genes; PPI, protein-protein interaction.

proliferation and invasion of breast cancer cells (42) and small cell lung cancer cells (43). In addition, a previous study has demonstrated that $\mathrm{ABCE} 1$ participates in the immune response of colon cancer (44). Consistent with these results, the present study demonstrated that $\mathrm{ABCE} 1$ was an upregulated hub DEG, and that high $\mathrm{ABCE} 1$ expression was associated with a high OS in patients with $\mathrm{CRC}$. These findings indicated that ABCE1 may be considered as a diagnostic and prognostic biomarker in CRC and may be used for targeted therapy of CRC.

TIMP1 is a soluble protein released from endometrium cells, fibroblasts and cancer cells, which has been demonstrated to be associated with prognosis in various types of carcinoma (45-47). Xiong et al (48) reported that TIMP1 is overexpressed in CRC. A recent study demonstrated that TIMP1 depletion can inhibit the proliferation, migration and invasion of colonic carcinoma cells, and inhibit the tumorigenesis and metastasis in CRC (49). In addition, TIMP1 overexpression is associated with focal adhesion kinase (FAK) activation. FAK is an upstream regulator of the PI3K-Akt signaling pathway, which is crucial in the cell survival pathway (50-52). Consistent with these studies, the present study reported that TIMP1 was up-regulated in CRC tissues samples compared with normal tissue samples, and that low TIMP1 expression was associated with a higher OS of patients with CRC. Therefore, TIMP1 may be considered as a potential biomarker that could be used to predict the clinical outcome of patients with CRC.

Abnormal AKR1B10 expression has been reported in numerous types of cancer, including breast cancer, endometrial cancer, oral squamous cell carcinoma and lung cancer $(35,53,54)$. AKR1B10, which is one member of the AKR superfamily, is an essential regulatory gene involved in the physiological function of intestinal tissue, including cell apoptosis, proliferation and migration (55). Previous studies have demonstrated that AKR1B10 expression is lower in CRC tissues compared with in normal colorectal tissues $(44,56)$. Furthermore, AKR1B10 is one of the direct transcriptional targets of the p53 gene $(34,57)$. Significantly, p53 is activated in cases of DNA damage, oncogene activation, anoxia and excessive proliferation (38). A previous study reported that the p53 mutation rate in CRC is $\sim 40 \%$ (56). In addition, survival analysis using the TCGA database has demonstrated that patients with CRC and high AKR1B10 expression have a significantly longer survival rate (58). Consistent with these findings, the present study demonstrated that AKR1B10 was down-regulated in CRC tissues compared with normal tissues, and that patients with CRC in the AKR1B10 high expression group had a higher OS compared with patients in the AKR1B10 low expression group. These results suggest 
Table III. GO analysis of the genes of each module in colorectal cancer tissues.

A, Module 1

\begin{tabular}{llcrc}
\hline Category & \multicolumn{1}{c}{ Term } & Count, n & Percentage & P-value \\
\hline GOTERM_BP_DIRECT & GO:0030198 'extracellular matrix organization' & 8 & 44.44 & $4.67 \times 10^{-10}$ \\
& GO:0030574 'collagen catabolic process' & 5 & 27.78 & $4.40 \times 10^{-7}$ \\
& GO:0022617 'extracellular matrix disassembly' & 5 & 22.78 & $8.82 \times 10^{-7}$ \\
& GO:0032355 'response to estradiol' & 4 & 22.22 & $9.91 \times 10^{-5}$ \\
GOTERM_CC_DIRECT & GO:0005615 'extracellular space' & 12 & 66.67 & $2.28 \times 10^{-11}$ \\
& GO:0005576 'extracellular region' & 12 & 66.67 & $1.38 \times 10^{-5}$ \\
GOTERM_MF_DIRECT & GO:0048407 'platelet-derived growth factor binding' & 3 & 16.67 & $5.22 \times 10^{-5}$ \\
& GO:0050840 'extracellular matrix binding' & 3 & 16.67 & $3.06 \times 10^{-4}$ \\
\hline
\end{tabular}

B, Module 2

\begin{tabular}{llrrr}
\hline Category & Term & Count, n & Percentage & P-value \\
\hline GOTERM_MF_DIRECT & GO:0016887 'ATPase activity' & 2 & 33.33 \\
& GO:0005515 'protein binding' & 5 & $3.05 \times 10^{-4}$ \\
& & 83.33 & $4.65 \times 10^{-4}$ \\
\hline
\end{tabular}

C, Module 3

\begin{tabular}{llrrr}
\hline Category & Term & Count, n & Percentage & P-value \\
\hline GOTERM_BP_DIRECT & GO:0051453 'regulation of intracellular pH' & 3 & 50 & $1.34 \times 10^{-5}$ \\
& GO:0015701 'bicarbonate transport' & 3 & 50 & $2.01 \times 10^{-5}$ \\
& GO:1902476 'chloride transmembrane transport' & 3 & 50 & $9.07 \times 10^{-5}$ \\
GOTERM_MF_DIRECT & GO:0005254 'chloride channel activity' & 3 & 50 & $6.00 \times 10^{-5}$ \\
\hline
\end{tabular}

$\mathrm{BP}$, biological process; $\mathrm{CC}$, cellular component; GO, Gene Ontology; MF, molecular function.

Table IV. Kyoto Encyclopedia of Genes and Genomes pathway analysis of the genes of each module in colorectal cancer tissues.

A, Module 1

\begin{tabular}{|c|c|c|c|c|}
\hline Term & Count, $\mathrm{n}$ & Percentage & P-value & Genes \\
\hline hsa04512: ECM-receptor interaction & 5 & 27.78 & $2.13 \times 10^{-5}$ & CD44, COL3A1, COL1A2, COL1A1, SPP1 \\
\hline $\begin{array}{l}\text { hsa04151: PI3K-Akt signaling } \\
\text { pathway }\end{array}$ & 7 & 38.89 & $3.17 \times 10^{-5}$ & $\begin{array}{l}\text { CCND1, COL3A1, MET, COL1A2, COL1A1, } \\
\text { MYC, SPP1 }\end{array}$ \\
\hline hsa04510: Focal adhesion & 6 & 33.33 & $3.61 \times 10^{-5}$ & $\begin{array}{l}\text { CCND1, COL3A1, MET, COL1A2, COL1A1, } \\
\text { SPP1 }\end{array}$ \\
\hline hsa05206: MicroRNAs in cancer & 3 & 33.33 & $1.70 \times 10^{-4}$ & $\begin{array}{l}\text { CCND1, CD44, PTGS2, SERPINB5, MET, } \\
\text { MYC }\end{array}$ \\
\hline
\end{tabular}

B, Module 3

\begin{tabular}{lcccc}
\hline Term & Count, $\mathrm{n}$ & Percentage & P-value & Genes \\
\hline hsa04972: Pancreatic secretion & 3 & 50 & $1.79 \times 10^{-4}$ & SLC26A3, CLCA4, SLC4A4 \\
\hline
\end{tabular}

ECM, extracellular matrix. 
Table V. Predictive values of clinical characteristics of patients with colorectal cancer and the four hub genes.

\begin{tabular}{|c|c|c|c|c|c|c|}
\hline \multirow[b]{2}{*}{ Variable } & \multirow[b]{2}{*}{ Group } & \multirow[b]{2}{*}{ Number } & \multicolumn{2}{|c|}{ Univariate analysis } & \multicolumn{2}{|c|}{ Multivariate analysis } \\
\hline & & & $95 \% \mathrm{CI}$ & P-value & $95 \% \mathrm{CI}$ & P-value \\
\hline Age (years) & $<60$ vs. $\geq 60$ & 173 vs. 424 & $1.043-2.483$ & 0.030 & $1.335-3.414$ & 0.002 \\
\hline Sex & Male vs. female & 322 vs. 275 & - & 0.818 & - & 0.566 \\
\hline UICC stage & I and II vs. III and IV & 338 vs. 259 & $2.094-4.591$ & $<0.001$ & $2.123-4.716$ & $<0.001$ \\
\hline TIMP1 & FPKM $<172.3$ vs. $\geq 172.3$ & 272 vs. 325 & $1.376-2.929$ & $<0.001$ & $1.019-2.302$ & 0.040 \\
\hline SLC4A4 & FPKM $<2.5$ vs. $\geq 2.5$ & 477 vs. 120 & $0.195-0.673$ & 0.001 & 0.189-0.709 & 0.003 \\
\hline AKR1B10 & FPKM $<2.5$ vs. $\geq 2.5$ & 207 vs. 390 & $0.365-0.739$ & $<0.001$ & $0.454-0.962$ & 0.030 \\
\hline ABCE1 & FPKM $<16.2$ vs. $\geq 16.2$ & 331 vs. 266 & $0.373-0.784$ & 0.001 & $0.414-0.930$ & 0.021 \\
\hline
\end{tabular}

ABCE1, ATP binding cassette subfamily E member 1; AKR1B10, aldo-keto reductase family 1 member B10; CI, confidence interval; FPKM, fragments per kilobase million; SLC4A4, solute carrier family 4 member 4; TIMP1, TIMP metallopeptidase inhibitor 1; UICC, Union for International Cancer Control.

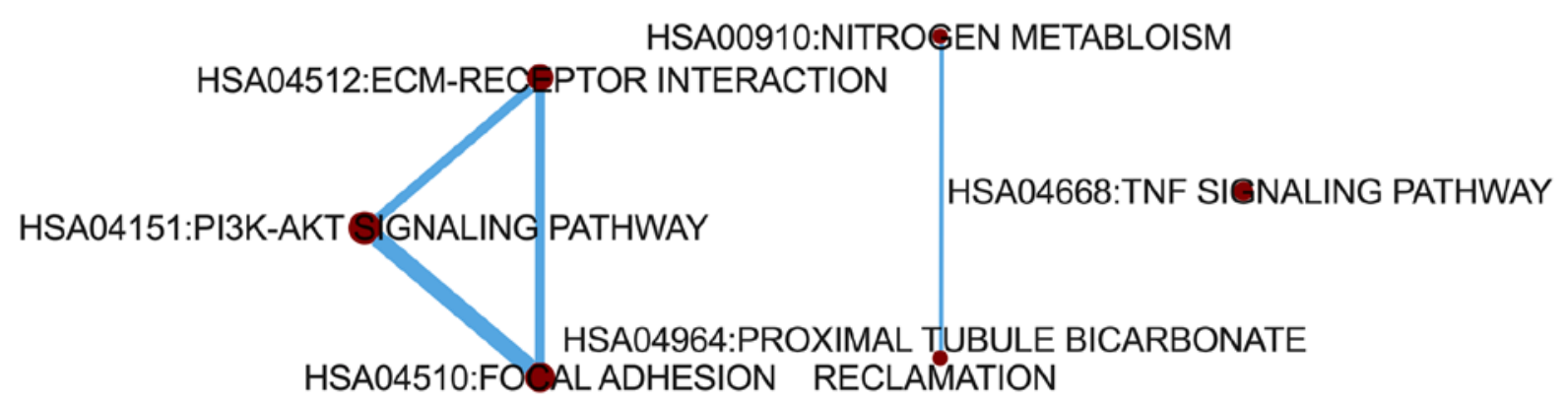

Figure 3. Crosstalk analysis for significant pathways. ECM, extracellular matrix; TNF, tumor necrosis factor.
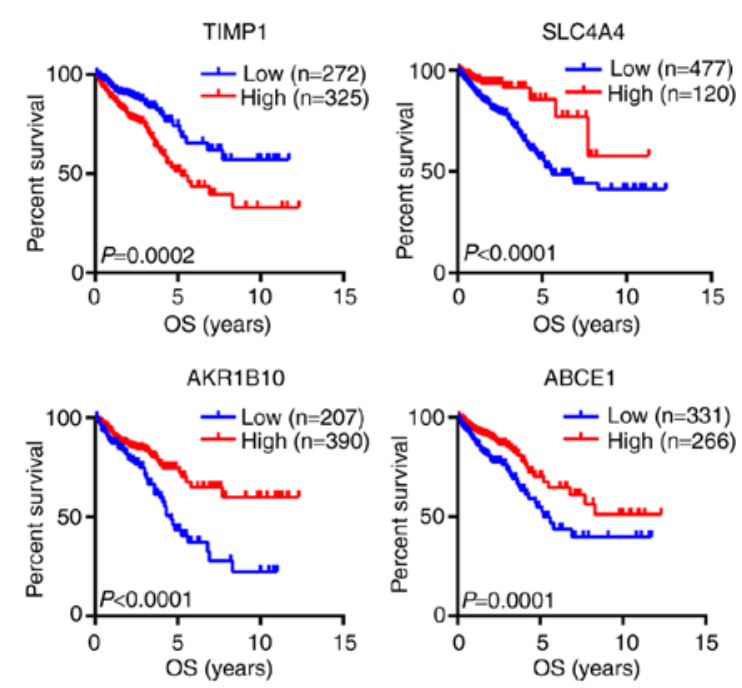

Figure 4. Kaplan-Meier OS curves according to the expression levels of TIMP1, SLC4A4, AKR1B10 and ABCE1. OS curves demonstrated that high TIMP1 expression and low SLC4A4/AKR1B10/ABCE1 expression were significantly associated with low OS in patients with colorectal cancer. ABCE1, ATP binding cassette subfamily E member 1; AKR1B10, aldo-keto reductase family 1 member B10; OS, overall survival; SLC4A4, solute carrier family 4 member 4; TIMP1, TIMP metallopeptidase inhibitor 1.

the importance of AKR1B10 in evaluating the prognosis of patients with CRC.

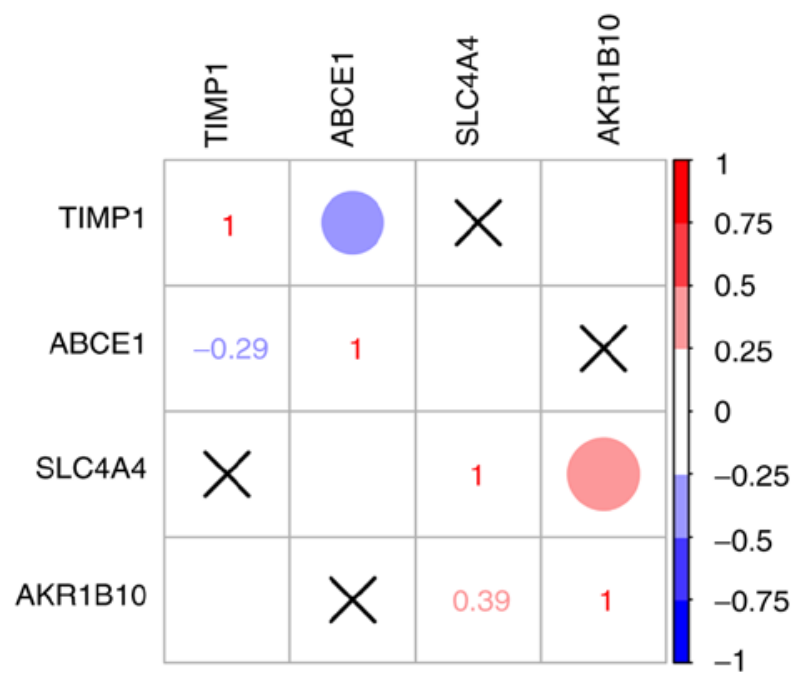

Figure 5. Correlations among the four hub genes (TIMP1, SLC4A4, AKR1B10 and ABCE1) evaluated using Spearman's correlation analysis based on Spearman's correlation coefficient $(|\mathrm{R}|<0.3)$ and $\mathrm{P}<0.05$. ABCE1, ATP binding cassette subfamily E member 1; AKR1B10, aldo-keto reductase family 1 member B10; SLC4A4, solute carrier family 4 member 4; TIMP1, TIMP metallopeptidase inhibitor 1 .

The results of the present study may have clinical significance for CRC; however, the current study presented certain 
limitations. Firstly, since the candidate prognosis-associated hub DEGs were detected using data from the GEO and TCGA databases, there was no validation for these genes based on the data generated in the present study. Secondly, the expression levels of the prognosis-associated hub DEGs were not validated using PCR, western blotting or other experimental methods. Thirdly, since the potential roles of AKR1B10, SLC4A4, ABCE1 and TIMP1 in CRC remain unknown, future investigations will determine the effects of these hub genes in CRC by using in vivo and in vitro experiments.

In conclusion, through GEO and TCGA data analyses, four hub DEGs (SLC4A4, ABCE1, AKR1B10 and TIMP1) were identified as being significantly associated with the OS of patients with CRC. These four genes may serve as novel independent prognostic biomarkers that could be used to predict the clinical outcomes of patients with CRC. However, further investigations using cancer cell lines and xenograft models are required in order to determine the underlying mechanisms of these four hub genes and their roles in the prognosis of CRC.

\section{Acknowledgements}

Not applicable.

\section{Funding}

The present study was supported by the Natural Science Foundation of Henan Province (grant no. 162300410040), the National Natural Science Foundation of China (grant no. 81301963) and the Outstanding Youth Science Foundation of Henan University (grant no. yqpy20140036).

\section{Availability of data and materials}

The datasets used and/or analyzed during the present study are available from the corresponding author on reasonable request.

\section{Authors' contributions}

GPD and LPW collected the data, analyzed the data and drafted the manuscript. YQW, XQR and SGZ designed the study and critically revised the manuscript. All authors read and approved the final version of the manuscript.

\section{Ethics approval and consent to participate}

Not applicable.

\section{Patient consent for publication}

Not applicable.

\section{Competing interests}

The authors declare that they have no competing interests.

\section{References}

1. Siegel R, Desantis C and Jemal A: Colorectal cancer statistics, 2014. CA Cancer J Clin 64: 104-117, 2014.
2. Bray F, Ferlay J, Soerjomataram I, Siegel RL, Torre LA and Jemal A: Global cancer statistics 2018: GLOBOCAN estimates of incidence and mortality worldwide for 36 cancers in 185 countries. CA Cancer J Clin 68: 394-424, 2018.

3. Leslie A and Steele RJ: Management of colorectal cancer. Postgrad Med J 78: 473-478, 2002.

4. Renkonen-Sinisalo L, Aarnio M, Mecklin JP and Järvinen HJ: Surveillance improves survival of colorectal cancer in patients with hereditary nonpolyposis colorectal cancer. Cancer Detect Prev 24: 137-142, 2000.

5. Siegel RL, Miller KD, Fedewa SA, Ahnen DJ, Meester RGS, Barzi A and Jemal A: Colorectal cancer statistics, 2017. CA Cancer J Clin 67: 177-193, 2017.

6. Chen W, Zheng R, Zeng H, Zhang S and He J: Annual report on status of cancer in China, 2011. Chin J Cancer Res 27: 2-12, 2015.

7. Derry MM, Raina K, Agarwal C and Agarwal R: Identifying molecular targets of lifestyle modifications in colon cancer prevention. Front Oncol 3: 119, 2013.

8. Sakai E, Fukuyo M, Ohata K, Matsusaka K, Doi N, Mano Y, Takane K, Abe H, Yagi K, Matsuhashi N, et al: Genetic and epigenetic aberrations occurring in colorectal tumors associated with serrated pathway. Int J Cancer 138: 1634-1644, 2016.

9. Mlcochova J, Faltejskova P, Nemecek R, Svoboda M and Slaby O: MicroRNAs targeting EGFR signalling pathway in colorectal cancer. J Cancer Res Clin Oncol 139: 1615-1624, 2013.

10. Takahashi T, Shigematsu H, Shivapurkar N, Reddy J, Zheng Y, Feng Z, Suzuk M, Nomura M, Augustus M, Yin J, et al: Aberrant promoter methylation of multiple genes during multistep pathogenesis of colorectal cancers. Int J Cancer 118: 924-931, 2006.

11. Schirripa M and Lenz HJ: Biomarker in Colorectal Cancer. Cancer J 22: 156-164, 2016.

12. Bustin SA and Dorudi S: Gene expression profiling for molecular staging and prognosis prediction in colorectal cancer. Expert Rev Mol Diagn 4: 599-607, 2004.

13. Kulasingam V and Diamandis EP: Strategies for discovering novel cancer biomarkers through utilization of emerging technologies. Nat Clin Pract Oncol 5: 588-599, 2008.

14. Nannini M, Pantaleo MA, Maleddu A, Astolfi A, Formica S and Biasco G: Gene expression profiling in colorectal cancer using microarray technologies: Results and perspectives. Cancer Treat Rev 35: 201-209, 2009.

15. Liang B, Li C and Zhao J: Identification of key pathways and genes in colorectal cancer using bioinformatics analysis. Med Oncol 33: 111, 2016.

16. Skrzypczak M, Goryca K, Rubel T, Paziewska A, Mikula M, Jarosz D, Pachlewski J, Oledzki J and Ostrowski J: Modeling oncogenic signaling in colon tumors by multidirectional analyses of microarray data directed for maximization of analytical reliability. PLoS One 5: e13091, 2010.

17. Condorelli DF, Spampinato G, Valenti G, Musso N, Castorina S and Barresi V: Positive caricature transcriptomic effects associated with broad genomic aberrations in colorectal cancer. Sci Rep 8: 14826, 2018.

18. Ryan BM, Zanetti KA, Robles AI, Schetter AJ, Goodman J, Hayes RB, Huang WY, Gunter MJ, Yeager M, Burdette L, et al: Germline variation in NCF4, an innate immunity gene, is associated with an increased risk of colorectal cancer. Int J Cancer 134: 1399-1407, 2014

19. Rich JN, Hans C, Jones B, Iversen ES, McLendon RE, Rasheed BK, Dobra A, Dressman HK, Bigner DD, Nevins JR and West M: Gene expression profiling and genetic markers in glioblastoma survival. Cancer Res 65: 4051-4058, 2005.

20. Simon R, Lam A, Li MC, Ngan M, Menenzes S and Zhao Y: Analysis of gene expression data using BRB-array tools. Cancer Inform 3: 11-17, 2007.

21. von Mering C, Huynen M, Jaeggi D, Schmidt S, Bork P and Snel B: STRING: A database of predicted functional associations between proteins. Nucleic Acids Res 31: 258-261, 2003.

22. Smoot ME, Ono K, Ruscheinski J, Wang PL and Ideker T: Cytoscape 2.8: New features for data integration and network visualization. Bioinformatics 27: 431-432, 2011.

23. Otte E and Rousseau R: Social network analysis: A powerful strategy, also for the information sciences. J Inform Sci 28: 441-453, 2002.

24. Rhrissorrakrai K and Gunsalus KC: MINE: Module identification in networks. BMC Bioinformatics 12: 192, 2011.

25. Ashburner M, Ball CA, Blake JA, Botstein D, Butler H, Cherry JM, Davis AP, Dolinski K, Dwight SS, Eppig JT, et al: Gene ontology: Tool for the unification of biology. The Gene Ontology Consortium. Nat Genet 25: 25-29, 2000. 
26. Ogata H, Goto S, Sato K, Fujibuchi W, Bono H and Kanehisa M: KEGG: Kyoto Encyclopedia of Genes and Genomes. Nucleic Acids Res 27: 29-34, 1999.

27. Huang da W, Sherman BT and Lempicki RA: Systematic and integrative analysis of large gene lists using DAVID bioinformatics resources. Nat Protoc 4: 44-57, 2009.

28. Merico D, Isserlin R, Stueker O, Emili A and Bader GD: Enrichment map: A network-based method for gene-set enrichment visualization and interpretation. PLoS One 5: e13984, 2010

29. Huang SH, Xu W, Waldron J, Siu L, Shen X, Tong L, Ringash J, Bayley A, Kim J, Hope A, et al: Refining American Joint Committee on Cancer/Union for International Cancer Control TNM stage and prognostic groups for human papillomavirus-related oropharyngeal carcinomas. J Clin Oncol 33: $836-845,2015$

30. Ramaswamyreddy SH and Smitha T: Microarray-based gene expression profiling for early detection of oral squamous cell carcinoma. J Oral Maxillofac Pathol 22: 293-295, 2018.

31. Salem H, Attiya G and El-Fishawy N: Classification of human cancer diseases by gene expression profiles. Appl Soft Comput 50: 124-134, 2017

32. Li G, Li X, Yang M, Xu L, Deng S and Ran L: Prediction of biomarkers of oral squamous cell carcinoma using microarray technology. Sci Rep 7: 42105, 2017.

33. Romero MF, Hediger MA, Boulpaep EL and Boron WF: Expression cloning and characterization of a renal electrogenic Na+/HCO3-cotransporter. Nature 387: 409-413, 1997.

34. Romero MF, Fulton CM and Boron WF: The SLC4 family of HCO 3-transporters. Pflugers Arch 447: 495-509, 2004.

35. Gomez-Rueda H, Palacios-Corona R, Gutiérrez-Hermosillo $\mathrm{H}$ and Trevino V: A robust biomarker of differential correlations improves the diagnosis of cytologically indeterminate thyroid cancers. Int J Mol Med 37: 1355-1362, 2016.

36. Chen S, Zhang L, Su Y and Zhang X: Screening potential biomarkers for colorectal cancer based on circular RNA chips. Oncol Rep 39: 2499-2512, 2018.

37. Zhao ZW, Fan XX, Yang LL, Song JJ, Fang SJ, Tu JF, Chen MJ, Zheng LY, Wu FZ, Zhang DK, et al: The identification of a common different gene expression signature in patients with colorectal cancer. Math Biosci Eng 16: 2942-2958, 2019.

38. Bisbal C, Martinand C, Silhol M, Lebleu B and Salehzada T: Cloning and Characterization of a RNase L Inhibitor A new component of the interferon-regulated 2-5A pathway. J Biol Chem 270: 13308-13317, 1995

39. Pestka S, Langer JA, Zoon KC and Samuel CE: Interferons and their actions. Annu Rev Biochem 56: 727-777, 1987.

40. Zheng D, Dai Y, Wang S and Xing X: MicroRNA-299-3p promotes the sensibility of lung cancer to doxorubicin through directly targeting ABCE1. Int J Clin Exp Pathol 8: 10072-10081, 2015.

41. Hlavata I, Mohelnikova-Duchonova B, Vaclavikova R, Liska V Pitule P, Novak P, Bruha J, Vycital O, Holubec L, Treska V, et al: The role of $\mathrm{ABC}$ transporters in progression and clinical outcome of colorectal cancer. Mutagenesis 27: 187-196, 2012.

42. Huang B, Zhou H, Lang X and Liu Z: siRNA-induced ABCE1 silencing inhibits proliferation and invasion of breast cancer cells. Mol Med Rep 10: 1685-1690, 2014.

43. Huang B, Gao Y, Tian D and Zheng M: A small interfering ABCE1-targeting RNA inhibits the proliferation and invasiveness of small cell lung cancer. Int J Mol Med 25: 687-693, 2010.

44. Shichijo S, Ishihara Y, Azuma K, Komatsu N, Higashimoto N, Ito $M$, Nakamura T, Ueno T, Harada $M$ and Itoh $\mathrm{K}$ : ABCE1, a member of ATP-binding cassette transporter gene, encodes peptides capable of inducing HLA-A2-restricted and tumor-reactive cytotoxic T lymphocytes in colon cancer patients. Oncol Rep 13: 907-913, 2005.
45. Wang YY, Li L, Zhao ZS and Wang HJ: Clinical utility of measuring expression levels of KAP1, TIMP1 and STC2 in peripheral blood of patients with gastric cancer. World J Surg Oncol 11: 81, 2013.

46. Bjerre C, Vinther L, Belling KC, Würtz S $\varnothing$, Yadav R, Lademann U, Rigina O, Do KN, Ditzel HJ, Lykkesfeldt AE, et al: TIMP1 overexpression mediates resistance of MCF-7 human breast cancer cells to fulvestrant and down-regulates progesterone receptor expression. Tumour Biol 34: 3839-3851, 2013.

47. Peng L, Yanjiao M, Ai-Guo W, Pengtao G, Jianhua L, Ju Y, Hongsheng O and Xichen Z: A fine balance between CCNL1 and TIMP1 contributes to the development of breast cancer cells. Biochem Biophys Res Commun 409: 344-349, 2011.

48. Xiong Y, You W, Wang R, Peng L and Fu Z: Prediction and validation of Hub Genes associated with colorectal cancer by integrating PPI network and gene expression data. Biomed Res Int 2017: 2421459, 2017.

49. Song G, Xu S, Zhang H, Wang Y, Xiao C, Jiang T, Wu L, Zhang T, Sun X,Zhong L, et al: TIMP1 is a prognostic marker for the progression and metastasis of colon cancer through FAK-PI3K/AKT and MAPK pathway. J Exp Clin Cancer Res 35: 148, 2016.

50. Kostourou V, Lechertier T, Reynolds LE, Lees DM, Baker M, Jones DT, Tavora B, Ramjaun AR, Birdsey GM, Robinson SD, et al: FAK-heterozygous mice display enhanced tumour angiogenesis. Nat Commun 4: 2020, 2013.

51. Oudart JB, Doué M, Vautrin A, Brassart B, Sellier C, Dupont-Deshorgue A, Monboisse JC, MaquartFX,Brassart-Pasco S and Ramont L: The anti-tumor NC1 domain of collagen XIX inhibits the FAK/PI3K/Akt/mTOR signaling pathway through $\alpha v \beta 3$ integrin interaction. Oncotarget 7: 1516-1528, 2016.

52. Kapur R, Cooper R,Zhang L and Williams DA: Cross-talk between alpha(4)beta(1)/alpha(5)beta(1) and c-Kit results in opposing effect on growth and survival of hematopoietic cells via the activation of focal adhesion kinase, mitogen-activated protein kinase, and Akt signaling pathways. Blood 97: 1975-1981, 2001.

53. Ko HH, Cheng SL, Lee JJ, Chen HM, Kuo MY and Cheng SJ: Expression of AKR1B10 as an independent marker for poor prognosis in human oral squamous cell carcinoma. Head Neck 39: 1327-1332, 2017.

54. Sinreih M, Štupar S, Čemažar L, Verdenik I, Frković Grazio S, Smrkolj S and Rižner TL: STAR and AKR1B10 are down-regulated in high-grade endometrial cancer. J Steroid Biochem Mol Bio 171: 43-53, 2017

55. Huang B, Gong X, Zhou H, Xiong F and Wang S: Depleting ABCE1 expression induces apoptosis and inhibits the ability of proliferation and migration of human esophageal carcinoma cells. Int J Clin Exp Pathol 7: 584-592, 2014.

56. Li Q, Shen F and Wang C: TUC338 promotes cell migration and invasion by targeting TIMP1 in cervical cancer. Oncol Lett 13: 4526-4532, 2017

57. Petitjean A, Mathe E, Kato S, Ishioka C, Tavtigian SV, Hainaut $P$ and Olivier M: Impact of mutant p53 functional properties on TP53 mutation patterns and tumor phenotype: Lessons from recent developments in the IARC TP53 database. Hum Mutat 28: 622-629, 2007

58. Taskoparan B, Seza EG, Demirkol S, Tuncer S, Stefek M, Gure AO and Banerjee S: Opposing roles of the aldo-keto reductases AKR1B1 and AKR1B10 in colorectal cancer. Cell Oncol (Dordr) 40: 563-578, 2017.

This work is licensed under a Creative Commons Attribution-NonCommercial-NoDerivatives 4.0 International (CC BY-NC-ND 4.0) License. 